\title{
Flaxion: a minimal extension to solve puzzles in the standard model
}

\author{
Yohei Ema, ${ }^{a}$ Koichi Hamaguchi, ${ }^{a, b}$ Takeo Moroi $^{a, b}$ and Kazunori Nakayama ${ }^{a, b}$ \\ ${ }^{a}$ Department of Physics, \\ The University of Tokyo, Tokyo 133-0033, Japan \\ ${ }^{b}$ Kavli Institute for the Physics and Mathematics of the Universe (Kavli IPMU), \\ University of Tokyo, Kashiwa 27r-8583, Japan \\ E-mail: ema@hep-th.phys.s.u-tokyo.ac.jp, \\ hama@hep-th.phys.s.u-tokyo.ac.jp, moroi@hep-th.phys.s.u-tokyo.ac.jp, \\ kazunori@hep-th.phys.s.u-tokyo.ac.jp
}

ABSTRACT: We propose a minimal extension of the standard model which includes only one additional complex scalar field, flavon, with flavor-dependent global U(1) symmetry. It not only explains the hierarchical flavor structure in the quark and lepton sector (including neutrino sector), but also solves the strong $\mathrm{CP}$ problem by identifying the CP-odd component of the flavon as the QCD axion, which we call flaxion. Furthermore, the flaxion model solves the cosmological puzzles in the standard model, i.e., origin of dark matter, baryon asymmetry of the universe, and inflation. We show that the radial component of the flavon can play the role of inflaton without isocurvature nor domain wall problems. The dark matter abundance can be explained by the flaxion coherent oscillation, while the baryon asymmetry of the universe is generated through leptogenesis.

Keywords: Beyond Standard Model, Cosmology of Theories beyond the SM, Global Symmetries, Quark Masses and SM Parameters

ARXIV EPRINT: 1612.05492 


\section{Contents}

1 Introduction 1

2 Flaxion 2

2.1 Model 2

2.2 Flavon interactions 4

2.3 Flaxion as QCD axion 5

2.4 Constraints on flaxion 6

3 Flaxion and flavon cosmology $\quad 8$

3.1 Flaxion as dark matter 8

$\begin{array}{lll}3.2 & \text { Isocurvature and domain wall problem } & 8\end{array}$

$\begin{array}{llr}3.3 & \text { Flavon inflation } & 9\end{array}$

$\begin{array}{ll}3.4 & \text { Suppression of isocurvature perturbation } \\ & 11\end{array}$

$\begin{array}{ll}3.5 & \text { Reheating after flavon inflation } \\ \end{array}$

4 Conclusions and discussion $\quad 13$

A Quark and lepton masses and mixings $\quad \mathbf{1 5}$

$\begin{array}{ll}\text { A.1 Quark and charged lepton masses and CKM matrix } & 15\end{array}$

$\begin{array}{ll}\text { A.2 Neutrino masses and mixing } & 16\end{array}$

\section{Introduction}

There are several puzzles in the standard model (SM) of particle physics, which may be solved by new physics models based on (spontaneously broken) symmetries. Although one may be able to introduce a new symmetry to solve each puzzle, it is desirable to have a unified picture of those symmetries from the point of view of simplicity and minimality, as we suggest in this paper.

One of the mysteries of the SM is the hierarchical flavor structure of the Yukawa couplings. The Froggatt-Nielsen mechanism is an attractive possibility to explain the quark/lepton mass hierarchy and their mixing matrices [1]. It introduces a new complex scalar field called flavon, whose vacuum expectation value (VEV) generates the SM Yukawa couplings. In this model a global Abelian flavor symmetry $\mathrm{U}(1)_{F}$ is imposed.

Another puzzle in the SM is the strong CP problem in the quantum chromo dynamics (QCD). The Peccei-Quinn (PQ) mechanism [2] utilizes a global U(1) symmetry, $\mathrm{U}(1)_{\mathrm{PQ}}$, to solve it; the pseudo Nambu-Goldstone boson associated with the spontaneous breaking of $\mathrm{U}(1)_{\mathrm{PQ}}$, called axion $[3,4]$, dynamically cancels the strong $\mathrm{CP}$ angle. Moreover, the PQ model explains the present dark matter (DM) abundance through the coherent oscillation of the axion field [5-7] if the breaking scale of $\mathrm{U}(1)_{\mathrm{PQ}}$ is at a relevant scale. It is also 
remarkable that the right-handed neutrinos can have large masses through the $\mathrm{U}(1)_{\mathrm{PQ}}$ breaking [8]. Thus tiny left-handed neutrino masses are naturally explained through the seesaw mechanism [9-11].

In this paper, we propose a new minimal extension of the $\mathrm{SM}$ in which the flavor $\mathrm{U}(1)_{F}$ and the $\mathrm{U}(1)_{\mathrm{PQ}}$ are unified. We introduce only one additional complex scalar field, flavon, charged under the global $\mathrm{U}(1)_{F}$ whose VEV naturally explains the Yukawa structure. As long as this $\mathrm{U}(1)_{F}$ is exact up to the QCD anomaly, its angular component remains nearly massless, which we call flaxion. Assuming that $\mathrm{U}(1)_{F}$ is anomalous under $\mathrm{SU}(3)_{C}$, the flaxion gets the potential after the QCD phase transition as ordinary axion and solves the strong CP problem. Similar possibility was already pointed out long ago in ref. [12] followed by several studies [13-21]. We use the minimality as our guiding principle and add only one complex scalar field to the SM. Then, with such an additional complex scalar field (as well as right-handed neutrinos), we show that it is possible to explain the followings: ${ }^{1}$ (1) Yukawa flavor structure, (2) strong CP problem, (3) neutrino masses and mixings, (4) dark matter, (5) baryon asymmetry, and (6) inflation. In particular, we point out that a successful inflation takes place by identifying the flavon field as the inflaton. By utilizing the idea of attractor inflation [25-28], we have a phenomenologically viable inflation with successful reheating consistent with leptogenesis [29] and without domain wall nor flaxion isocurvature fluctuation problems.

We emphasize that our model is more economical than other axion models. In the KSVZ axion model [30, 31] heavy vector-like quarks are necessary, while in the DFSZ axion model $[32,33]$ we need two Higgs doublets. In this sense, our model is economical: addition of only one new scalar field is sufficient to explain the flavor structure, solve the strong CP problem and provide a good DM candidate.

This paper is organized as follows. In section 2, we present our model and derive flavon/flaxion coupling to the SM particles. Experimental constraints, in particular flavorviolating neutral current (FCNC) processes mediated by the flaxion, are also summarized. In section 3, cosmological aspects of the flaxion model is discussed. There we show that the flavon field acts as the inflaton through the attractor-type mechanism for flattening the potential without domain wall nor isocurvature problems. We conclude in section 4 with several remarks.

\section{Flaxion}

\subsection{Model}

The model we consider is described by the following Yukawa terms in the Lagrangian:

$$
\begin{aligned}
-\mathcal{L}= & y_{i j}^{d}\left(\frac{\phi}{M}\right)^{n_{i j}^{d}} \bar{Q}_{i} H d_{R j}+y_{i j}^{u}\left(\frac{\phi}{M}\right)^{n_{i j}^{u}} \bar{Q}_{i} \widetilde{H} u_{R j}+y_{i j}^{l}\left(\frac{\phi}{M}\right)^{n_{i j}^{l}} \bar{L}_{i} H l_{R j} \\
& +y_{i \alpha}^{\nu}\left(\frac{\phi}{M}\right)^{n_{i \alpha}^{\nu}} \bar{L}_{i} \widetilde{H} N_{R \alpha}+\frac{1}{2} y_{\alpha \beta}^{N}\left(\frac{\phi}{M}\right)^{n_{\alpha \beta}^{N}} M \overline{N_{R \alpha}^{c}} N_{R \beta}+\text { h.c. },
\end{aligned}
$$

\footnotetext{
${ }^{1}$ A similar approach was made in refs. [22-24] in the framework of KSVZ axion model, although they did not address the flavor structure.
} 
where $M$ is a mass scale corresponding to the cut-off scale of this model. Here $Q_{i}, u_{R i}$, $d_{R i}, L_{i}, e_{R i}, N_{R \alpha}(i=1-3)$ denote the left-handed quark doublet, right-handed up-type quark, right-handed down-type quark, left-handed lepton doublet, right-handed charged lepton and right-handed neutrino, respectively. $H$ denotes the SM Higgs doublet, and $\widetilde{H}=i \sigma_{2} H^{*}$. Finally, $\phi$ is a complex scalar field, called flavon, whose VEV $\langle\phi\rangle \equiv v_{\phi}$ gives rise to the SM Yukawa couplings [1]. The hierarchy of the Yukawa coupling constants is explained by the smallness of $\epsilon$ defined as

$$
\epsilon \equiv \frac{v_{\phi}}{M}
$$

We assume all $y_{i j} \sim \mathcal{O}(1)$ and $\epsilon \sim 0.2$ to explain the hierarchical structure of the Yukawa matrix (see appendix A). For a while we do not specify the number of right-handed neutrinos. The minimal number required to reproduce the experimental results is two $(\alpha=1,2)[34,35]$, while we do not exclude the possibility of three right-handed neutrinos $(\alpha=1-3)$. After $\phi$ and $H$ get VEVs, the mass matrices are given by

$$
m_{i j}^{d}=y_{i j}^{d} \epsilon^{n_{i j}^{d}} v_{\mathrm{EW}}, \quad m_{i j}^{u}=y_{i j}^{u} \epsilon^{n_{i j}^{u}} v_{\mathrm{EW}}, \quad m_{i j}^{l}=y_{i j}^{l} \epsilon^{n_{i j}^{l}} v_{\mathrm{EW}},
$$

where $\langle H\rangle \equiv v_{\text {EW }}=174 \mathrm{GeV}^{2}$

This model possesses a global chiral $\mathrm{U}(1)$ symmetry, which we denote by $\mathrm{U}(1)_{F}$, under which the flavon is assumed to have a charge +1 and the SM Higgs is neutral. Denoting the $\mathrm{U}(1)_{F}$ charges of the SM quarks and leptons as $q_{Q_{i}}, q_{u_{i}}$ etc., we have the following relations:

$$
\begin{aligned}
& n_{i j}^{u}=q_{Q_{i}}-q_{u_{j}}, \\
& n_{i j}^{d}=q_{Q_{i}}-q_{d_{j}}, \\
& n_{i j}^{l}=q_{L_{i}}-q_{l_{j}}, \\
& n_{i \alpha}^{\nu}=q_{L_{i}}-q_{N_{\alpha}} \\
& n_{\alpha \beta}^{N}=-q_{N_{\alpha}}-q_{N_{\beta}} .
\end{aligned}
$$

An example for generating the desired quark and lepton masses and the CKM matrix is ${ }^{3}$

$$
\left(\begin{array}{ccc}
q_{Q_{1}} & q_{Q_{2}} & q_{Q_{3}} \\
q_{u} & q_{c} & q_{t} \\
q_{d} & q_{s} & q_{b}
\end{array}\right)=\left(\begin{array}{ccc}
3 & 2 & 0 \\
-5 & -1 & 0 \\
-4 & -3 & -3
\end{array}\right),
$$

and

$$
\left(\begin{array}{ccc}
q_{L_{1}} & q_{L_{2}} & q_{L_{3}} \\
q_{e} & q_{\mu} & q_{\tau}
\end{array}\right)=\left(\begin{array}{ccc}
1 & 0 & 0 \\
-8 & -5 & -3
\end{array}\right) .
$$

\footnotetext{
${ }^{2}$ The Higgs boson may naturally have mass of $\sim M$ in this framework. The fine-tuning issue to obtain the electroweak scale is not addressed in the present study.

${ }^{3}$ The charges of the left-handed fields, $q_{Q_{i}}$ and $q_{L_{i}}$, are chosen to approximately reproduce the CKM and MNS matrices. The other charges, $q_{u_{i}}, q_{d_{i}}, q_{l_{i}}$ are determined by $n_{i i}^{f} \simeq \log \left(m_{i}^{f} / m_{i}^{t}\right) / \log \epsilon$ with $\epsilon \simeq 0.23$. (See appendix A).
} 
That means

$$
n_{i j}^{u}=\left(\begin{array}{lll}
8 & 4 & 3 \\
7 & 3 & 2 \\
5 & 1 & 0
\end{array}\right), \quad n_{i j}^{d}=\left(\begin{array}{lll}
7 & 6 & 6 \\
6 & 5 & 5 \\
4 & 3 & 3
\end{array}\right), \quad n_{i j}^{l}=\left(\begin{array}{lll}
9 & 6 & 4 \\
8 & 5 & 3 \\
8 & 5 & 3
\end{array}\right)
$$

Note that for this charge assignment on the lepton doublets, the large $\nu_{\mu}-\nu_{\tau}$ mixing of the neutrino sector is obtained independently of the charges of the right-handed neutrinos [3638]. (See appendix A.2).

\subsection{Flavon interactions}

Now let us see the flavon interactions. Expanding the flavon and Higgs as

$$
\phi=v_{\phi}+\frac{1}{\sqrt{2}}(s+i a), \quad H=\left(\begin{array}{c}
0 \\
v_{\mathrm{EW}}+\frac{h}{\sqrt{2}}
\end{array}\right),
$$

the quark and charged lepton sectors of the Lagrangian (2.1) are written as

$$
-\mathcal{L}=\sum_{f=u, d, l}\left[m_{i j}^{f}\left(1+\frac{h}{\sqrt{2} v_{\mathrm{EW}}}\right)+\frac{m_{i j}^{f} n_{i j}^{f}(s+i a)}{\sqrt{2} v_{\phi}}\right] \overline{f_{L i}} f_{R j}+\text { h.c. }
$$

The mass term and Higgs Yukawa interactions are simultaneously diagonalized by the biunitary transformation

$$
f_{R_{j}} \equiv U_{j i}^{f} f_{R_{i}}^{\prime}, \quad f_{L_{i}} \equiv V_{i j}^{f} f_{L_{j}}^{\prime}, \quad\left(V^{f \dagger} m^{f} U^{f}\right)_{i j}=m_{i}^{f} \delta_{i j},
$$

but the terms involving the flavon interaction cannot be diagonalized:

$$
-\mathcal{L}=\sum_{f=u, d, l}\left[m_{i}^{f}\left(1+\frac{h}{\sqrt{2} v_{\mathrm{EW}}}\right) \overline{f_{L i}^{\prime}} f_{R i}^{\prime}+\kappa_{i j}^{f} \frac{s+i a}{\sqrt{2} v_{\phi}} \overline{f_{L i}^{\prime}} f_{R j}^{\prime}\right]+\text { h.c. }
$$

where the matrix $\kappa_{i j}^{f}$ is given by

$$
\kappa_{i j}^{f} \equiv V_{i k}^{f \dagger}\left(m_{k n}^{f} n_{k n}^{f}\right) U_{n j}^{f} .
$$

Thus the flavon and pseudo-scalar flavon mediate FCNC processes [12, 48-52]. The interaction of the pseudo-scalar flavon is then written as

$$
-\mathcal{L}=\frac{i a}{\sqrt{2} v_{\phi}} \sum_{f=u^{\prime}, d^{\prime}, l^{\prime}}\left[\left(\kappa_{\mathrm{H}}^{f}\right)_{i j} \bar{f}_{i} \gamma_{5} f_{j}+\left(\kappa_{\mathrm{AH}}^{f}\right)_{i j} \bar{f}_{i} f_{j}\right],
$$

where $\kappa_{\mathrm{H}}^{f}=\left(\kappa^{f}+\kappa^{f \dagger}\right) / 2$ and $\kappa_{\mathrm{AH}}^{f}=\left(\kappa^{f}-\kappa^{f \dagger}\right) / 2$ are Hermitian and anti-Hermitian parts of $\kappa^{f}$, respectively. Here it may be useful to rewrite the matrices $\kappa^{f}$ in a simple form. First note that, the factor $m_{k n}^{f} n_{k n}^{f}$ is expressed in a matrix form as

$$
\begin{aligned}
& m_{k n}^{f} n_{k n}^{f}=\left(\widehat{q}_{Q} m^{f}-m^{f} \widehat{q}_{f}\right)_{k n}, \quad f=u, d \\
& m_{k n}^{l} n_{k n}^{l}=\left(\widehat{q}_{L} m^{f}-m^{l} \widehat{q}_{l}\right)_{k n},
\end{aligned}
$$


where $\left(\widehat{q}_{X}\right)_{i j}=q_{X_{i}} \delta_{i j}$ are diagonal matrices. Then we obtain

$$
\kappa_{i j}^{f}=\left(V^{f \dagger} \widehat{q}_{Q} V^{f}\right)_{i j} m_{j}^{f}-m_{i}^{f}\left(U^{f \dagger} \widehat{q}_{f} U^{f}\right)_{i j},
$$

and

$$
\begin{aligned}
\left(\kappa_{\mathrm{H}}^{f}\right)_{i j} & =\frac{1}{2}\left(V^{f \dagger} \widehat{q}_{Q} V^{f}-U^{f \dagger} \widehat{q}_{f} U^{f}\right)_{i j}\left(m_{j}^{f}+m_{i}^{f}\right), \\
\left(\kappa_{\mathrm{AH}}^{f}\right)_{i j} & =\frac{1}{2}\left(V^{f \dagger} \widehat{q}_{Q} V^{f}+U^{f \dagger} \widehat{q}_{f} U^{f}\right)_{i j}\left(m_{j}^{f}-m_{i}^{f}\right) .
\end{aligned}
$$

for $f=u, d$. Expressions for $\kappa^{l}$ are obtained by replacing $\widehat{q}_{Q}$ with $\widehat{q}_{L}$.

\subsection{Flaxion as QCD axion}

The interaction between the pseudo-scalar flavon and quarks (2.17) yields the effective axion-gluon-gluon interaction through the triangle anomaly diagram. The effective interaction is given by

$$
\mathcal{L}=\frac{g_{s}^{2}}{32 \pi^{2}} \frac{a}{f_{a}} G_{\mu \nu}^{a} \widetilde{G}^{\mu \nu a}
$$

where

$$
f_{a} \equiv \frac{\sqrt{2} v_{\phi}}{N_{\mathrm{DW}}}=\frac{\sqrt{2} \epsilon M}{N_{\mathrm{DW}}}
$$

with the domain-wall number

$$
N_{\mathrm{DW}}=\operatorname{Tr}\left(2 \widehat{q}_{Q}-\widehat{q}_{u}-\widehat{q}_{d}\right)=\operatorname{Tr}\left(n^{u}+n^{d}\right),
$$

which corresponds to the number of the minima of the potential. In the model of section 2.1, $N_{\text {DW }}=26$. As is well known, after taking the QCD instanton effects into account, the interaction (2.23) results in the axion potential to cancel the strong CP angle at the potential minimum. Therefore, we can regard the pseudo-scalar flavon $a$ as the axion that solves the strong CP problem via the PQ mechanism. We call $a$ as the flaxion. The relation between the flaxion mass and the PQ scale is the same as the ordinary QCD axion [39]:

$$
m_{a} \simeq 6 \times 10^{-6} \mathrm{eV}\left(\frac{10^{12} \mathrm{GeV}}{f_{a}}\right) .
$$

Except that it has a relatively large domain wall number, its cosmological property is the same as the ordinary invisible QCD axion. In particular, the coherent oscillation of the flaxion can be a good DM candidate. We will discuss the cosmology of flaxion and the flavon in section 3 .

The flaxion-photon coupling is also important for low-energy phenomenology. The effective Lagrangian is given by

$$
\mathcal{L}=g_{a \gamma} \frac{e^{2}}{32 \pi^{2}} \frac{a}{f_{a}} F_{\mu \nu} \widetilde{F}^{\mu \nu}
$$


where [39]

$$
g_{a \gamma} \equiv \frac{2}{N_{\mathrm{DW}}} \sum_{f=u, d, l}\left[N_{f} \operatorname{Tr}\left(n^{f}\right)\left(q_{f}^{(\mathrm{em})}\right)^{2}\right]-\frac{2(4+z)}{3(1+z)},
$$

with $z \equiv m_{u} / m_{d} \simeq 0.56, q_{f}^{(\mathrm{em})}$ the electromagnetic charge of quarks and leptons, and $N_{f}=3$ (1) for quarks (leptons). For the model presented in section 2.1, we have $g_{a \gamma}=$ $113 / 39-1.95 \simeq 0.95$. Thus the prospects for the detection of the flaxion DM are similar to the KSVZ and DFSZ axion model [40-43].

\subsection{Constraints on flaxion}

Phenomenological consequences of the flaxion are similar to the DFSZ axion, except that the flaxion has FCNC interactions with the quarks and leptons. Here we briefly summarize constraints coming from the flavor-violating process induced by the flaxion and also astrophysical constraints.

The most stringent bound on $f_{a}$ may come from the process $K^{+} \rightarrow \pi^{+} a$ mediated by the second term of (2.17). In order to evaluate the matrix element of such a process, we adopt $\left\langle\pi\left(p_{\pi}\right)\left|\bar{s} \gamma^{\mu} d(x)\right| K\left(p_{K}\right)\right\rangle \simeq F_{1}\left(\left(p_{K}-p_{\pi}\right)^{2}\right) e^{-i\left(p_{K}-p_{\pi}\right) \cdot x}\left(p_{K}+p_{\pi}\right)^{\mu}$, with $F_{1}(0) \simeq 1$, which holds in the exact SU(3) flavor symmetry limit. Then, the matrix element is given by $\mathcal{M}=\frac{\left(\kappa_{\mathrm{AH}}^{d}\right)_{12}}{\sqrt{2} v_{\phi}}\left\langle\pi\left(p_{\pi}\right)|\bar{s} d| K\left(p_{K}\right)\right\rangle=\frac{\left(\kappa_{\mathrm{AH}}^{d}\right)_{12}}{\sqrt{2} v_{\phi}} \frac{m_{K}^{2}-m_{\pi}^{2}}{m_{s}-m_{d}}$, where we have also used the following relation: $\partial_{\mu}\left\langle\pi\left(p_{\pi}\right)\left|\bar{s} \gamma^{\mu} d\right| K\left(p_{K}\right)\right\rangle=\left(m_{s}-m_{d}\right)\left\langle\pi\left(p_{\pi}\right)|\bar{s} d| K\left(p_{K}\right)\right\rangle$. Consequently, the decay rate is evaluated as

$$
\Gamma\left(K^{+} \rightarrow \pi^{+} a\right)=\frac{m_{K}^{3}}{32 \pi v_{\phi}^{2}}\left(1-\frac{m_{\pi}^{2}}{m_{K}^{2}}\right)^{3}\left|\frac{\left(\kappa_{\mathrm{AH}}^{d}\right)_{12}}{m_{s}-m_{d}}\right|^{2},
$$

which gives

$$
\operatorname{Br}\left(K^{+} \rightarrow \pi^{+} a\right) \simeq 3 \times 10^{-10}\left(\frac{10^{10} \mathrm{GeV}}{f_{a}}\right)^{2}\left(\frac{26}{N_{\mathrm{DW}}}\right)^{2}\left|\frac{\left(\kappa_{\mathrm{AH}}^{d}\right)_{12}}{m_{s}-m_{d}}\right|^{2} .
$$

Comparing with the current experimental bound, $\operatorname{Br}\left(K^{+} \rightarrow \pi^{+} a\right) \lesssim 7.3 \times 10^{-11}$ [44], the bound on $f_{a}$ is given by

$$
f_{a} \gtrsim 2 \times 10^{10} \mathrm{GeV}\left(\frac{26}{N_{\mathrm{DW}}}\right)\left|\frac{\left(\kappa_{\mathrm{AH}}^{d}\right)_{12}}{m_{s}}\right| .
$$

Notice that, because $q_{Q_{1}}-q_{Q_{2}}=1$ in order to realize realistic flavor structure (see appendix A), $\left|\left(\kappa_{\mathrm{AH}}^{d}\right)_{12} / m_{s}\right| \sim O(\epsilon)$ (or larger, depending on the $\mathrm{U}(1)_{F}$ charges of the quarks) assuming no accidental cancellation. In the near future, it is expected that the NA62 experiment [45] will improve the measurement of $K^{+} \rightarrow \pi^{+} \bar{\nu} \nu$ (and $K^{+} \rightarrow \pi^{+} a$ ), improving the bound on $f_{a}$.

There are also lepton-flavor violating processes mediated by the flaxion. Note that processes including double flaxion vertices such as $\mu-e$ conversion or $\mu \rightarrow 3 e$ are highly suppressed. On the other hand, the decay of muon including the flaxion as a final state 
might give a stringent bound. The three body decay $\mu \rightarrow e a \gamma[46-48]$ might be the best to constrain the flaxion coupling to the lepton sector. The constraint reads $\operatorname{Br}(\mu \rightarrow$ ea $\gamma) \lesssim$ $1.1 \times 10^{-9}$, which is translated to [46]

$$
f_{a} \gtrsim 1 \times 10^{8} \mathrm{GeV}\left(\frac{26}{N_{\mathrm{DW}}}\right)\left|\frac{\left(\kappa_{\mathrm{AH}}^{l}\right)_{12}}{m_{\mu}}\right| .
$$

On the other hand, the observation of SN1987A event at Kamiokande constrains the flaxion-nucleon coupling, so that the duration of supernova does not change significantly. The flaxion-nucleon coupling is given by

$$
\mathcal{L}=\sum_{N=p, n} \frac{C_{N} m_{N}}{f_{a}} i a \bar{N} \gamma_{5} N,
$$

where

$$
\begin{aligned}
C_{p} & \simeq\left(\frac{\left(\kappa_{\mathrm{H}}^{u}\right)_{11}}{m_{u} N_{\mathrm{DW}}}-\frac{1}{1+z}\right) \Delta u+\left(\frac{\left(\kappa_{\mathrm{H}}^{d}\right)_{11}}{m_{d} N_{\mathrm{DW}}}-\frac{z}{1+z}\right) \Delta d, \\
C_{n} & \simeq\left(\frac{\left(\kappa_{\mathrm{H}}^{u}\right)_{11}}{m_{u} N_{\mathrm{DW}}}-\frac{1}{1+z}\right) \Delta d+\left(\frac{\left(\kappa_{\mathrm{H}}^{d}\right)_{11}}{m_{d} N_{\mathrm{DW}}}-\frac{z}{1+z}\right) \Delta u,
\end{aligned}
$$

with $\Delta f$ being the spin content of the nucleon: $S_{\mu} \Delta f \equiv\left\langle N\left|\bar{f} \gamma_{\mu} \gamma_{5} f\right| N\right\rangle$. They are given by $\Delta u=0.85$ and $\Delta d=-0.41$ [53], resulting in $C_{p} \simeq-0.4$ and $\left|C_{n}\right| \ll\left|C_{p}\right|$ for $N_{\text {DW }} \gg 1$. The constraint reads [54]

$$
\frac{f_{a}}{\left|C_{N}\right|} \gtrsim 1 \times 10^{9} \mathrm{GeV}
$$

which is weaker than the constraint from $K^{+} \rightarrow \pi^{+} a$. It is a striking property of the flaxion, which has flavor-violating couplings, that the most stringent lower bound on the PQ scale comes from the flavor physics, not from the SN1987A. The flaxion-electron coupling is also constrained by the observations of white dwarf stars so that the cooling of the white dwarf stars due to the flaxion emission does not affect the observed luminosity functions of white dwarf stars too much. The constraint reads [55]

$$
f_{a} \gtrsim 7 \times 10^{7} \mathrm{GeV}\left(\frac{26}{N_{\mathrm{DW}}}\right)\left|\frac{\left(\kappa_{\mathrm{H}}^{l}\right)_{11}}{m_{e}}\right| .
$$

Observations of horizontal branch stars and red-giant stars also put similar constraints on the flaxion-electron coupling [53].

Let us also comment on the possible constraint from nucleon decay caused by gaugeinvariant baryon- and lepton-number violating higher dimensional operators [56, 57]. If the cutoff scale of these operators are of order $M$, these operators are schematically written as

$$
\mathcal{L} \sim \frac{Q Q Q L}{M^{2}}, \frac{\text { uude }}{M^{2}}, \frac{Q Q u e}{M^{2}}, \frac{Q L u d}{M^{2}},
$$

which are multiplied by some powers of $\phi / M$ to be consistent with $\mathrm{U}(1)_{F}$ symmetry. Due to the suppression factor of powers of $\epsilon=v_{\phi} / M$, the effective cutoff scale of these 
operators can be much higher than $M$. For the charge assignments of (2.9) and (2.10), the most dangerous operator is the last one in (2.38), which is suppressed only by $\epsilon^{5}$ for the first generation quarks and leptons. Therefore, the effective cutoff scale of this operator is $M_{\text {eff }} \sim \epsilon^{-2.5} M \sim 40 \times M$ and hence we need $M \gtrsim 5 \times 10^{14} \mathrm{GeV}$ to avoid the too rapid proton decay [58]. This is roughly consistent with the phenomenologically preferred value $M \sim 10^{14}-10^{17} \mathrm{GeV}$, as shown in the next section. One should also note that this suppression factor crucially depends on the $\mathrm{U}(1)_{F}$ charge assignments on the quarks and leptons. As shown in appendix A.1, we have a freedom of constant shift of all the $\left(q_{Q_{i}}, q_{u_{i}}, q_{d_{i}}\right)$ without affecting $n_{i j}^{u}$ and $n_{i j}^{d}$. Using this freedom, it is possible to suppress all of the operators in (2.38) further. Since the Lagrangian (2.1) depends only on the combination $n_{i j}$, all the phenomenological constraints discussed so far, except for the nucleon decay, remain intact with such a shift of $\mathrm{U}(1)_{F}$ charges.

\section{$3 \quad$ Flaxion and flavon cosmology}

\subsection{Flaxion as dark matter}

Let us discuss cosmological consequences of the present model [59]. As in the case of ordinary QCD axion, the flaxion starts to oscillate around the minimum of the potential. Its present density is given by [60]

$$
\Omega_{a} h^{2}=0.18 \theta_{i}^{2}\left(\frac{f_{a}}{10^{12} \mathrm{GeV}}\right)^{1.19}
$$

where $\theta_{i}$ denotes the initial misalignment angle which takes the value $0 \leq \theta_{i}<2 \pi$. Thus, the flaxion oscillation can be dark matter for $f_{a} \sim O\left(10^{12}-10^{15}\right) \mathrm{GeV}$, assuming $\theta_{i} \simeq$ $O(0.01-1)$.

As discussed in the previous section, the decay constant of the flaxion is related to the parameters in the flavon potential. For $N_{\mathrm{DW}}=26$ and $\epsilon \sim 0.2$, for example, the flaxion dark matter is realized when $v_{\phi} \sim O\left(10^{13}-10^{16}\right) \mathrm{GeV}$ and $M \sim O\left(10^{14}-10^{17}\right) \mathrm{GeV}$.

\subsection{Isocurvature and domain wall problem}

Since the domain wall number is larger than unity, one may require that the $\mathrm{U}(1)_{F}$ symmetry be spontaneously broken during inflation to avoid the serious domain wall problem. In this case there is a stringent constraint on the inflation energy scale so that the flaxion does not acquire too large isocurvature fluctuations. The recent constraint from the Planck result reads $\sqrt{\mathcal{P}_{S} / \mathcal{P}_{\zeta}} \lesssim 0.18$ with $\mathcal{P}_{\zeta} \simeq 2.2 \times 10^{-9}$, where $\mathcal{P}_{S}$ and $\mathcal{P}_{\zeta}$ are the dimensionless power spectrum of the (uncorrelated) DM isocurvature and curvature perturbations, respectively [61]. If the flavon field settles down to the potential minimum during inflation, we have

$$
\mathcal{P}_{S} \simeq\left(\frac{H_{\mathrm{inf}}}{\pi f_{a} \theta_{i}}\right)^{2}\left(\frac{\Omega_{a} h^{2}}{\Omega_{\mathrm{CDM}} h^{2}}\right)^{2}
$$


where $\Omega_{a} h^{2}$ is given by eq. (3.1) and $\Omega_{\mathrm{CDM}} h^{2} \simeq 0.12$. Thus the inflationary scale is bounded as

$$
H_{\text {inf }} \lesssim 3 \times 10^{7} \mathrm{GeV} \theta_{i}^{-1}\left(\frac{10^{12} \mathrm{GeV}}{f_{a}}\right)^{0.19}
$$

Notice that this constraint is based on the assumption that the flavon already settles down to its potential minimum during inflation. However, the dynamics of the flavon field can be non-trivial during and after inflation and it can significantly modify the constraint. Below we see that the flavon itself can play the role of inflaton, avoiding this isocurvature bound.

\subsection{Flavon inflation}

So far we have assumed that the inflaton sector is independent of the SM + flavon sector. More interestingly, it may be possible to identify the flavon itself as the inflaton. ${ }^{4}$ First of all, one should note that large field inflation in which $\varphi \equiv \sqrt{2} \operatorname{Re}(\phi)$ rolls down from $\varphi \gg v_{\phi}$ would be dangerous, since during the reheating stage the flavon passes through the origin $\varphi=0$ many times and it leads to the nonthermal symmetry restoration through the parametric resonant enhancement of the flaxion field [63-66]. Thus the domain wall problem arises after the QCD phase transition in such a case. On the other hand, the smallfield inflation in which $\varphi$ rolls down from near the origin toward the potential minimum may be possible. Although there is no domain wall problem in this case, the flavon self coupling constant needs to be very small and also the flaxion isocurvature perturbation tends to be too large because it is enhanced due to the smallness of $\varphi$ during inflation.

Here we propose a nonminimal large-field inflation model which avoids these difficulties. The idea is to extend the flavon kinetic term to effectively flatten the potential at large field value $[67,68]$. In an extreme case in which the kinetic term has a pole at some field value, the effective potential becomes completely flat around the pole after the canonical normalization, and it leads to a class of large field inflation with best-fit value of the scalar spectral index [25-28].

Here, we adopt the following Lagrangian:

$$
\mathcal{L}=-\frac{|\partial \phi|^{2}}{\left(1-\frac{|\phi|^{2}}{\Lambda^{2}}\right)^{2}}-\lambda_{\phi}\left(|\phi|^{2}-v_{\phi}^{2}\right)^{2} .
$$

After the canonical normalization, the flavon potential may be rewritten as

$$
\mathcal{L}=-\frac{(\partial \widetilde{\varphi})^{2}}{2}-\lambda_{\phi}\left[\Lambda^{2} \tanh ^{2}\left(\frac{\widetilde{\varphi}}{\sqrt{2} \Lambda}\right)-v_{\phi}^{2}\right]^{2}
$$

where

$$
\frac{\varphi}{\sqrt{2} \Lambda} \equiv \tanh \left(\frac{\widetilde{\varphi}}{\sqrt{2} \Lambda}\right)
$$

Thus the potential is flat for $\widetilde{\varphi} \gg \Lambda$ and inflation can take place there. If $v_{\phi}<\Lambda<\sqrt{2} v_{\phi}$, the potential height at large field limit is lower than that at the origin (see figure 1), hence

\footnotetext{
${ }^{4}$ A flavon inflation was considered in ref. [62] in a different context.
} 


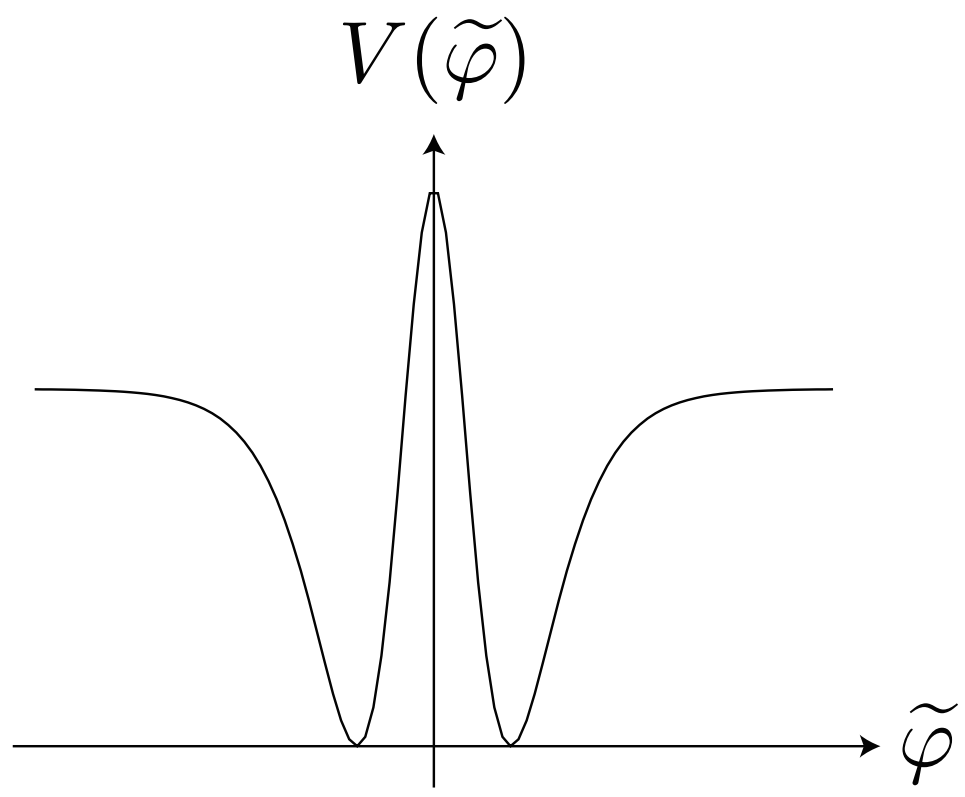

Figure 1. Schematic picture of the flavon potential for successful inflation.

the flavon does not pass through the origin after inflation. Thus there is no domain wall problem in this case. Note that the potential minimum in terms of $\widetilde{\varphi}$ is

$$
\frac{\langle\widetilde{\varphi}\rangle}{\sqrt{2}}=\Lambda \tanh ^{-1}\left(\frac{v_{\phi}}{\Lambda}\right) .
$$

As we will discuss in the following, $\Lambda$ is found to be of the same order of $v_{\phi}$ in the parameter region of our interest, and hence $\langle\widetilde{\varphi}\rangle \sim O\left(v_{\phi}\right)$.

We also note here that, due to the structure of the kinetic term in the present model, the field $a$ is not canonically normalized. The canonically-normalzed flaxion field around the vacuum is $\tilde{a} \equiv a / \Delta$, where

$$
\Delta \equiv 1-v_{\phi}^{2} / \Lambda^{2}
$$

Thus, the flaxion interactions (as well as the decay rate) given in the previous section should take account of the correction factor $\Delta$. For the case of our interest, however, $\Delta \sim O(1)$ and hence the discussion given in the previous section is qualitatively unchanged.

We can analyze the slow-roll inflation dynamics as usual [69]. The flavon field value during inflation is calculated as

$$
\widetilde{\varphi}_{N} \simeq \frac{\Lambda}{\sqrt{2}} \ln \left(\frac{16 N_{e} M_{P}^{2}}{\Lambda^{2}-v_{\phi}^{2}}\right),
$$

where $N_{e} \sim 50-60$ denotes the $e$-folding number at which the present horizon scale exits the horizon. The scalar spectral index $n_{s}$ and the tensor-to-scalar ratio $r$ are given by

$$
n_{s} \simeq 1-\frac{2}{N_{e}}, \quad r \simeq \frac{4}{N_{e}^{2}}\left(\frac{\Lambda}{M_{P}}\right)^{2} .
$$


Thus the scalar spectral index falls into the Planck best-fit region while the tensor-to-scalar ratio is too small to be detected. The dimensionless power spectrum of the curvature perturbation is given by

$$
\mathcal{P}_{\zeta} \simeq \frac{N_{e}^{2}}{6 \pi^{2}} \frac{\lambda_{\phi}\left(\Lambda^{2}-v_{\phi}^{2}\right)^{2}}{\Lambda^{2} M_{P}^{2}} .
$$

In order to reproduce the observed magnitude of the curvature perturbation, $\mathcal{P}_{\zeta} \simeq 2.2 \times$ $10^{-9}[61]$,

$$
\lambda_{\phi} \simeq 3 \times 10^{-2}\left(\frac{50}{N_{e}}\right)^{2}\left(\frac{10^{14} \mathrm{GeV}}{\Lambda}\right)^{2}\left(\frac{\Lambda^{2}}{\Lambda^{2}-v_{\phi}^{2}}\right)^{2} .
$$

In order for $\lambda_{\phi}$ to be in the perturbative range, we must have $\Lambda \gtrsim 10^{13} \mathrm{GeV}$, meaning $f_{a} \sim \Lambda / N_{\text {DW }} \gtrsim 5 \times 10^{11} \mathrm{GeV}$. This is consistent with the scale inferred from the flaxion DM density (3.1). The inflation scale is given by

$$
H_{\mathrm{inf}} \simeq 5 \times 10^{8} \mathrm{GeV}\left(\frac{\Lambda}{10^{14} \mathrm{GeV}}\right) .
$$

\subsection{Suppression of isocurvature perturbation}

Here we show that the isocurvature perturbation of the flaxion is highly suppressed due to the peculiar structure of the kinetic term. We parametrize the complex flavon field as $\phi=\varphi e^{i \Theta} / \sqrt{2}$. Then, the action for the phase $\Theta$ is given by

$$
\mathcal{L}=\frac{\Lambda^{2}}{4} \sinh ^{2}\left(\frac{\sqrt{2} \widetilde{\varphi}}{\Lambda}\right)(\partial \Theta)^{2} .
$$

Since $\widetilde{\varphi}$ slow-rolls during inflation, we may regard the prefactor in (3.14) as a constant. Then, the canonically normalized field during inflation is given by

$$
\tilde{a}_{\text {inf }}=\frac{\Lambda}{\sqrt{2}} \sinh \left(\frac{\sqrt{2} \widetilde{\varphi}}{\Lambda}\right) \Theta .
$$

The canonical field $\tilde{a}_{\text {inf }}$ acquires long-wavelength fluctuations of $H_{\text {inf }} / 2 \pi$ during inflation, and hence the original phase $\Theta$ fluctuates as

$$
\mathcal{P}_{\delta \Theta} \simeq \frac{2 H_{\mathrm{inf}}^{2}}{\pi^{2} \Lambda^{2}} \exp \left(-\frac{2 \sqrt{2} \widetilde{\varphi}_{N}}{\Lambda}\right) \simeq \frac{H_{\mathrm{inf}}^{2}}{128 \pi^{2} \Lambda^{2}}\left(\frac{\Lambda^{2}-v_{\phi}^{2}}{N_{e} M_{P}^{2}}\right)^{2},
$$

where $\mathcal{P}_{\delta \Theta}$ is the power spectrum of $\delta \Theta$. (Here, we have used $\widetilde{\varphi} \gg \Lambda$ during inflation.) Since it is related to the fluctuation of the initial misalignment angle as $\delta \theta_{i}=N_{\mathrm{DW}} \delta \Theta$, the ratio of the $\mathrm{DM}$ isocurvature perturbation to the curvature perturbation is estimated as

$$
\frac{\mathcal{P}_{S}}{\mathcal{P}_{\zeta}} \simeq \frac{R_{a}^{2} N_{\mathrm{DW}}^{2}}{64 \theta_{i}^{2} N_{e}^{4}} \frac{\left(\Lambda^{2}-v_{\phi}^{2}\right)^{2}}{M_{P}^{4}},
$$

which is highly suppressed. ${ }^{5}$ Thus, the observational bound is safely satisfied and the flaxion can be the dominant component of DM.

\footnotetext{
${ }^{5}$ In this scenario, the spectrum of the flaxion isocurvature fluctuation is blue. However, even at the smallest scale the isocurvature perturbation is small enough.
} 


\subsection{Reheating after flavon inflation}

Finally let us discuss the reheating after flavon inflation. There are mainly three decay modes of the flavon: decay into right-handed neutrinos, decay into Higgs bosons and decay into flaxions. Other decay modes are suppressed either by the loop factor or the final state fermion masses.

The flavon partial decay rate into the right-handed neutrino pair is given by

$$
\Gamma\left(\widetilde{\varphi} \rightarrow N_{R} N_{R}\right) \simeq \sum_{\alpha \beta} \frac{\left|y_{\alpha \beta}^{N} n_{\alpha \beta}^{N} \epsilon^{n_{\alpha \beta}^{N}-1}\right|^{2}}{32 \pi} \Delta^{2} m_{\varphi}
$$

where the flavon mass around the potential minimum is given by

$$
m_{\varphi}^{2}=4 \lambda_{\phi} v_{\phi}^{2} \Delta^{2}
$$

Note that $m_{\varphi} \sim 3 \times 10^{13} \mathrm{GeV}\left(v_{\phi} / \Lambda\right)$ is almost independent of the overall scale $\Lambda$. Here we have assumed that the flavon is heavier than the right-handed neutrino: $4 \lambda_{\phi} \Delta^{2} \gtrsim$ $\left(y_{\alpha \alpha}^{N} \epsilon^{n_{\alpha \alpha}^{N}-1}\right)^{2}$. The partial decay rate of flavon into the Higgs bosons depend on the additional potential term ${ }^{6}$

$$
V=\lambda_{\phi H}|\phi|^{2}|H|^{2}
$$

We find the partial decay rate into the Higgs boson pair as

$$
\Gamma(\widetilde{\varphi} \rightarrow H H) \simeq \frac{\Delta^{2}}{8 \pi} \frac{\lambda_{\phi H}^{2} v_{\phi}^{2}}{m_{\varphi}} \simeq \frac{1}{32 \pi} \frac{\lambda_{\phi H}^{2}}{\lambda_{\phi}} m_{\varphi}
$$

where we have taken account of the four real degrees of freedom in the SM Higgs doublet. ${ }^{7}$ On the other hand, the flavon partial decay rate into the flaxion pair is given by

$$
\Gamma(\widetilde{\varphi} \rightarrow a a) \simeq \frac{\Delta^{2}}{32 \pi} \frac{m_{\varphi}^{3}}{v_{\phi}^{2}} \simeq \frac{\lambda_{\phi}}{8 \pi} \Delta^{4} m_{\varphi} .
$$

Thus the total decay width of the flavon is

$$
\Gamma_{\widetilde{\varphi}} \simeq\left(\sum_{\alpha, \beta}\left|y_{\alpha \beta}^{N} n_{\alpha \beta}^{N} \epsilon^{n_{\alpha \beta}^{N}-1}\right|^{2} \frac{\Delta^{2}}{4}+\frac{\lambda_{\phi H}^{2}}{4 \lambda_{\phi}}+\lambda_{\phi} \Delta^{4}\right) \frac{m_{\varphi}}{8 \pi} .
$$

This is typically much larger than $H_{\text {inf }}$ and hence the reheating is completed almost instantaneously after inflation. Thus the reheating temperature, $T_{\mathrm{R}}$, can be

\footnotetext{
${ }^{6}$ This term potentially leads to the vacuum decay through the resonant enhancement of the Higgs fluctuation [70-73]. However, the same term along with large VEV of $\phi$ can ensure the absolute stability of the Higgs potential $[74,75]$. Note also that there must be a large bare mass term of the Higgs to cancel the flavon-induced mass term so that it obtains the electroweak scale VEV.

${ }^{7}$ This coupling radiatively affects the flavon potential. If it is substantially large and $\lambda_{\phi}$ is too small, the flavon potential can be dominated by the radiatively-induced effective potential.
} 
as high as $10^{12}-10^{14} \mathrm{GeV}$ in our scenario. Flaxions are thermalized through interactions with Higgs and right-handed neutrinos and there is no problem of flaxion dark radiation overproduction.

Lastly let us discuss thermal leptogenesis in the present scenario. The final baryon asymmetry through the leptogenesis from the decay of right-handed neutrinos is given by $[76]$

$$
\frac{n_{B}}{s} \simeq \epsilon_{1} \kappa_{f} \frac{28}{79}\left(\frac{n_{N_{1}}}{s}\right)_{\mathrm{th}} \simeq 1.3 \times 10^{-3} \epsilon_{1} \kappa_{f},
$$

where $\left(n_{N_{1}} / s\right)_{\text {th }}$ is the abundance of the right-handed neutrino in thermal equilibrium, $\epsilon_{1}$ denotes the lepton asymmetry generated by per right-handed neutrino decay and $\kappa_{f}$ denotes the efficiency factor. The asymmetry parameter is calculated as

$$
\epsilon_{1}=\frac{3}{16 \pi} \frac{m_{N_{1}} m_{\nu_{3}}}{v_{\mathrm{EW}}^{2}} \delta_{\mathrm{eff}} \simeq 1 \times 10^{-4}\left(\frac{m_{N_{1}}}{10^{12} \mathrm{GeV}}\right)\left(\frac{m_{\nu 3}}{0.05 \mathrm{eV}}\right) \delta_{\mathrm{eff}},
$$

where $\delta_{\text {eff }}$ is the effective CP angle which satisfies $\delta_{\text {eff }} \leq 1$ for $m_{N_{1}} \ll m_{N_{2(3)}}[77,78]{ }^{8}$ On the other hand, the efficiency factor $\kappa_{f}$ crucially depends on the effective neutrino mass $\widetilde{m}_{\nu 1} \equiv \sum_{k}\left|\epsilon^{n k 1} y_{k 1}^{\nu}\right|^{2} v_{\mathrm{EW}}^{2} / m_{N_{1}}$. In the present scenario, it is roughly given by $\widetilde{m}_{\nu 1} \sim$ $\sum_{k} \epsilon^{2 q_{L_{k}}} v_{\mathrm{EW}}^{2} / M \sim m_{\nu_{3}}$. (See appendix A.) This corresponds to a so-called strong washout regime $\left(\widetilde{m}_{\nu 1} \gtrsim m_{*} \simeq 1 \times 10^{-3} \mathrm{eV}\right)$, where the efficiency factor is approximately given by $\kappa_{f} \sim 0.02 \times\left(\widetilde{m}_{\nu 1} / 0.01 \mathrm{eV}\right)^{-1.1}[76]$. For $\widetilde{m}_{\nu 1} \sim m_{\nu_{3}} \sim 0.05 \mathrm{eV}$, we obtain $\kappa_{f} \sim$ $3 \times 10^{-3}$. Therefore, the observed baryon asymmetry $n_{B} / s \simeq 9 \times 10^{-11}$ can be obtained for $m_{N_{1}} \sim O\left(10^{12}\right) \mathrm{GeV} .{ }^{9}$ This can be obtained, for instance, by taking $q_{N_{1}}=1-5$ for $M \sim O\left(10^{14}-10^{17}\right) \mathrm{GeV}$.

\section{Conclusions and discussion}

We have shown that a simple QCD axion model in which $\mathrm{U}(1)_{\mathrm{PQ}}$ is identified with Abelian flavor symmetry $\mathrm{U}(1)_{F}$ solves and explains puzzles in the SM. The model contains only one additional complex scalar and right-handed neutrinos. Inflation can successfully happen without domain wall nor isocurvature problems.

Here are some remarks. In this paper, we assume that there is only one Higgs doublet. Although this is a minimal choice, if there are additional Higgs doublets, we can assign the $\mathrm{U}(1)_{F}$ charges to the Higgses so that $N_{\mathrm{DW}}=1$. As an example, we consider a two Higgs doublet model (2HDM) [87] with the following Yukawa interactions (the so-called type-II or type-Y $2 \mathrm{HDM})$ :

$$
-\mathcal{L}=y_{i j}^{d}\left(\frac{\phi}{M}\right)^{n_{i j}^{d}} \bar{Q}_{i} H_{d} d_{R j}+y_{i j}^{u}\left(\frac{\phi}{M}\right)^{n_{i j}^{u}} \bar{Q}_{i} H_{u} u_{R j} .
$$

\footnotetext{
${ }^{8}$ If the mass of $N_{1}$ is degenerated with $N_{2}$, the asymmetry is enhanced [79-83]. This can happen in our case if $\mathrm{U}(1)_{F}$ charges of right-handed neutrinos are the same.

${ }^{9}$ For this mass scale, none of the charged lepton Yukawa coupling is in equilibrium, and the flavor effect [84-86] can be neglected.
} 
If we assign the $\mathrm{U}(1)_{F}$ charges $q_{H_{u}}$ and $q_{H_{d}}$ on $H_{u}$ and $H_{d}$ respectively, we obtain

$$
\begin{aligned}
& n_{i j}^{d}=q_{Q_{i}}-q_{d_{j}}-q_{H_{d}}, \\
& n_{i j}^{u}=q_{Q_{i}}-q_{u_{j}}-q_{H_{u}} .
\end{aligned}
$$

We may keep $n_{i j}^{f}$ the same as those in (2.11) by shifting the charges of the right-handed quarks as $q_{f_{i}} \rightarrow q_{f_{i}}-q_{H_{f}} .{ }^{10}$ Then, the domain wall number is given by

$$
N_{\mathrm{DW}}=\left|\operatorname{Tr}\left(2 \widehat{q}_{Q}-\widehat{q}_{u}-\widehat{q}_{d}\right)\right|=\left|26+3\left(q_{H_{u}}+q_{H_{d}}\right)\right| .
$$

Thus, the domain wall number is $N_{\mathrm{DW}}=1$ if we take $q_{H_{u}}+q_{H_{d}}=-9$. In this case, there is no cosmological domain wall problem even if the PQ symmetry is restored during inflation, as long as $f_{a}<(4.6-7.2) \times 10^{10} \mathrm{GeV}$ [88] and hence there can be a variety of cosmological scenarios.

Although the minimality is lost, it is also easy to embed the theory into the supersymmetry (SUSY) framework. We can just interpret the Lagrangian (4.1) as the superpotential written by the chiral superfields. Since there are two Higgs doublets in minimal SUSY SM, we can choose the $\mathrm{U}(1)_{F}$ charges so that $N_{\mathrm{DW}}=1$ as just shown above. In this case, the $\mu$-term can be generated by the superpotetnial $W \sim(\phi / M)^{9} M H_{u} H_{d}$, which may be compatible with high-scale SUSY scenario in which the soft mass scale is $O(100-1000) \mathrm{TeV}$. The potential of the flavon can be generated by introducing $\bar{\phi}$ and also a "stabilizer field" $X$, which have $\mathrm{U}(1)_{F}$ charges -1 and 0 respectively, and assume the superpotential

$$
W=\lambda X\left(\phi \bar{\phi}-v_{\phi}^{2}\right)
$$

After they get soft SUSY breaking masses, they are stabilized at $\phi \sim \bar{\phi} \sim v_{\phi}$. Since $\bar{\phi}$ is oppositely charged under $\mathrm{U}(1)_{F}$, it cannot directly couple to SM Yukawa terms. Note that, with the present assignments of $\mathrm{U}(1)_{F}$ charges, off-diagonal elements of the squark mass matrix are not suppressed enough to avoid SUSY flavor problem if the mass scale of the SUSY particiles is around TeV. Such a problem can be solved by high-scale SUSY or flavorblind mediation model (like gauge mediation). (Otherwise one may adopt a different flavor symmetry to suppress the off-diagonal elements of the sfermion mass matrix.) Cosmology of this class of models will be non-trivial due to the presence of sflaxion and flaxino which appear in the flavon supermultiplet, although the detailed investigation is beyond the scope of this paper.

\section{Acknowledgments}

We thank Natsumi Nagata for helpful discussion. This work was supported by the Grantin-Aid for Scientific Research on Scientific Research A (No.26247038 [KH], No.26247042 $[\mathrm{KN}]$, No.16H02189 [KH]), Scientific Research C (No.26400239[TM]), Young Scientists B (No.26800121 [KN], No.26800123 [KH]) and Innovative Areas (No.26104001 [KH],

\footnotetext{
${ }^{10}$ If $\tan \beta \equiv\left\langle H_{u}\right\rangle /\left\langle H_{d}\right\rangle$ is not of order unity, the ratio of the overall normalization of $n_{i j}^{d}$ and $n_{i j}^{u}$ can be much different than (2.11).
} 
No.26104009 [KH and KN], No.15H05888 [KN], No.16H06490 [TM]), and by World Premier International Research Center Initiative (WPI Initiative), MEXT, Japan. The work of YE was supported in part by JSPS Research Fellowships for Young Scientists and by the Program for Leading Graduate Schools, MEXT, Japan.

\section{A Quark and lepton masses and mixings}

\section{A.1 Quark and charged lepton masses and CKM matrix}

For general $\mathrm{U}(1)_{F}$ charge assignments on the quark fields

$$
\left(\begin{array}{ccc}
q_{Q_{1}} & q_{Q_{2}} & q_{Q_{3}} \\
q_{u} & q_{c} & q_{t} \\
q_{d} & q_{s} & q_{b}
\end{array}\right)
$$

with assumption $q_{Q_{i}} \geq q_{Q_{j}} \geq 0$ and $q_{f_{i}} \leq q_{f_{j}} \leq 0$ for $i<j$, the quark mass matrix (normalized by $v_{\mathrm{EW}}$ ) is expressed and decomposed as

$$
\begin{aligned}
& m_{i j}^{d} \sim \epsilon^{n_{i j}^{d}} \sim V^{d} \operatorname{diag}\left(m_{d}\right) U^{d \dagger} \\
& \sim\left(\begin{array}{ccc}
1 & \epsilon^{q_{Q_{1}}-q_{Q_{2}}} & \epsilon^{q_{Q_{1}}-q_{Q_{3}}} \\
\epsilon^{q_{Q_{1}}-q_{Q_{2}}} & 1 & \epsilon^{q_{2}-q_{Q_{3}}} \\
\epsilon^{q_{Q_{1}}-q_{Q_{3}}} & \epsilon^{q_{Q_{2}}-q_{Q_{3}}} & 1
\end{array}\right)\left(\begin{array}{ccc}
\epsilon^{q_{Q_{1}}-q_{d}} & 0 & 0 \\
0 & \epsilon^{q_{2}-q_{s}} & 0 \\
0 & 0 & \epsilon^{q_{Q_{3}}-q_{b}}
\end{array}\right)\left(\begin{array}{ccc}
1 & \epsilon^{q_{s}-q_{d}} & \epsilon^{q_{b}-q_{d}} \\
\epsilon^{q_{s}-q_{d}} & 1 & \epsilon^{q_{b}-q_{s}} \\
\epsilon^{q_{b}-q_{d}} & \epsilon^{q_{b}-q_{s}} & 1
\end{array}\right), \\
& m_{i j}^{u} \sim \epsilon^{n_{i j}^{u}} \sim V^{u} \operatorname{diag}\left(m_{u}\right) U^{u \dagger} \\
& \sim\left(\begin{array}{ccc}
1 & \epsilon^{q_{Q_{1}}-q_{Q_{2}}} & \epsilon^{q_{Q_{1}}-q_{Q_{3}}} \\
\epsilon^{q_{Q_{1}}-q_{Q_{2}}} & 1 & \epsilon^{q_{Q_{2}}-q_{Q_{3}}} \\
\epsilon^{Q_{Q_{1}}-q_{Q_{3}}} & \epsilon^{q_{Q_{2}}-q_{Q_{3}}} & 1
\end{array}\right)\left(\begin{array}{ccc}
\epsilon^{q_{Q_{1}}-q_{u}} & 0 & 0 \\
0 & \epsilon^{q_{Q_{2}}-q_{c}} & 0 \\
0 & 0 & \epsilon^{q_{Q_{3}}-q_{t}}
\end{array}\right)\left(\begin{array}{ccc}
1 & \epsilon^{q_{c}-q_{u}} & \epsilon^{q_{t}-q_{u}} \\
\epsilon^{q_{c}-q_{u}} & 1 & \epsilon^{q_{t}-q_{c}} \\
\epsilon^{q_{t}-q_{u}} & \epsilon^{q_{t}-q_{c}} & 1
\end{array}\right) .
\end{aligned}
$$

Thus the CKM matrix is given by

$$
V_{\mathrm{CKM}}=V^{u \dagger} V^{d} \sim\left(\begin{array}{ccc}
1 & \epsilon^{q_{Q_{1}}-q_{Q_{2}}} & \epsilon^{q_{Q_{1}}-q_{Q_{3}}} \\
\epsilon^{q_{Q_{1}}-q_{Q_{2}}} & 1 & \epsilon^{q_{Q_{2}}-q_{Q_{3}}} \\
\epsilon^{q_{Q_{1}}-q_{Q_{3}}} & \epsilon^{q_{Q_{2}}-q_{Q_{3}}} & 1
\end{array}\right),
$$

which depends only on the charges of the left-handed quarks. Taking account of $O(1)$ Yukawa couplings, it well reproduces observed values of the CKM matrix elements for

$$
\left(\begin{array}{lll}
q_{Q_{1}} & q_{Q_{2}} & q_{Q_{3}}
\end{array}\right)=\left(q_{Q_{3}}+3 q_{Q_{3}}+2 q_{Q_{3}}\right),
$$

and $\epsilon \simeq 0.23$. Charges of right-handed quarks are chosen so that the quark mass eigenvalues are consistent with observed values:

$$
\begin{array}{lll}
q_{Q_{1}}-q_{d}=7, & q_{Q_{2}}-q_{s}=5, & q_{Q_{3}}-q_{b}=3, \\
q_{Q_{1}}-q_{u}=8, & q_{Q_{2}}-q_{c}=3, & q_{Q_{3}}-q_{t}=0 .
\end{array}
$$

Still we have a degree of freedom to choose $q_{Q_{3}}$, corresponding to the overall constant shift of $\left(q_{Q_{i}}, q_{u_{i}}, q_{d_{i}}\right){ }^{11}$ A particular example with $q_{Q_{3}}=0$ is given in (2.9).

\footnotetext{
${ }^{11}$ In other words, we can arbitrarily add baryon charges to the $\mathrm{U}(1){ }_{F}$ charges.
} 
Similarly, for general $\mathrm{U}(1)_{F}$ charge assignments on the leptons

$$
\left(\begin{array}{ccc}
q_{L_{1}} & q_{L_{2}} & q_{L_{3}} \\
q_{e} & q_{\mu} & q_{\tau}
\end{array}\right)
$$

with assumption $q_{L_{i}} \geq q_{L_{j}} \geq 0$ and $q_{f_{i}} \leq q_{f_{j}} \leq 0$ for $i<j$, the charged lepton mass matrix (normalized by $v_{\mathrm{EW}}$ ) is decomposed as

$$
\begin{aligned}
m_{i j}^{l} & \sim \epsilon^{n_{i j}^{l}} \sim V^{l} \operatorname{diag}\left(m_{l}\right) U^{l \dagger} \\
& \sim\left(\begin{array}{ccc}
1 & \epsilon^{q_{L_{1}}-q_{L_{2}}} & \epsilon^{q_{L_{1}}-q_{L_{3}}} \\
\epsilon^{q_{L_{1}}-q_{L_{2}}} & 1 & \epsilon^{q_{L_{2}}-q_{L_{3}}} \\
\epsilon^{q_{L_{1}}-q_{L_{3}}} & \epsilon^{q_{L_{2}}-q_{L_{3}}} & 1
\end{array}\right)\left(\begin{array}{ccc}
\epsilon^{q_{L_{1}}-q_{e}} & 0 & 0 \\
0 & \epsilon^{q_{L_{2}}-q_{\mu}} & 0 \\
0 & 0 & \epsilon^{q_{L_{3}}-q_{\tau}}
\end{array}\right)\left(\begin{array}{ccc}
1 & \epsilon^{q_{\mu}-q_{e}} & \epsilon^{q_{\tau}-q_{e}} \\
\epsilon^{q_{\mu}-q_{e}} & 1 & \epsilon^{q_{\tau}-q_{\mu}} \\
\epsilon^{q_{\tau}-q_{e}} & \epsilon^{q_{\tau}-q_{\mu}} & 1
\end{array}\right) .
\end{aligned}
$$

The observed charged lepton masses are reproduced for

$$
\left(q_{L_{1}} q_{L_{2}} q_{L_{3}}\right)=\left(q_{e}+9 q_{\mu}+5 q_{\tau}+3\right) .
$$

The charges of left-handed leptons are partly constrained from the neutrino mass matrix, as shown below.

\section{A.2 Neutrino masses and mixing}

First let us consider the minimal case of two right-handed neutrinos: $N_{\alpha}(\alpha=1,2)$. For general $\mathrm{U}(1)_{F}$ charge assignments on right-handed neutrinos $\left(q_{N_{1}} q_{N_{2}}\right)$, the Dirac- and Majorana-mass matrices of neutrinos are given by

$$
\left(m_{D}^{\nu}\right)_{i \alpha} \sim v_{\mathrm{EW}}\left(\begin{array}{cc}
\epsilon^{q_{L_{1}}-q_{N_{1}}} & \epsilon^{q_{L_{1}}-q_{N_{2}}} \\
\epsilon^{q_{L_{2}}-q_{N_{1}}} & \epsilon^{q_{L_{2}}-q_{N_{2}}} \\
\epsilon^{q_{L_{3}}-q_{N_{1}}} & \epsilon^{q_{L_{3}}-q_{N_{2}}}
\end{array}\right), \quad\left(m^{N}\right)_{\alpha \beta} \sim M\left(\begin{array}{cc}
\epsilon^{-2 q_{N_{1}}} & \epsilon^{-q_{N_{1}}-q_{N_{2}}} \\
\epsilon^{-q_{N_{1}}-q_{N_{2}}} & \epsilon^{-2 q_{N_{2}}}
\end{array}\right) .
$$

According to the seesaw mechanism, after integrating out heavy right-handed neutrinos, we obtain the following light neutrino mass matrix:

$$
m_{i j}^{\nu}=m_{D}^{\nu} \cdot\left(m^{N}\right)^{-1} \cdot\left(m_{D}^{\nu}\right)^{T} \sim \frac{v_{\mathrm{EW}}^{2}}{M}\left(\begin{array}{ccc}
\epsilon^{2 q_{L_{1}}} & \epsilon^{q_{L_{1}}+q_{L_{2}}} & \epsilon^{q_{L_{1}}+q_{L_{3}}} \\
\epsilon_{L_{1}+q_{L_{2}}}^{q_{2}} & \epsilon^{2 q_{L_{2}}} & \epsilon^{q_{L_{2}}+q_{L_{3}}} \\
\epsilon^{q_{L_{1}}+q_{L_{3}}} & \epsilon^{q_{L_{2}}+q_{L_{3}}} & \epsilon^{2 q_{L_{3}}}
\end{array}\right) .
$$

It is independent of the charges of right-handed neutrinos. Note that since the matrix $m^{N}$ is rank $2, m_{i j}^{\nu}$ must contain one zero eigenvalue. It is diagonalized as

$$
\begin{aligned}
m_{i j}^{\nu} & \sim U^{\nu} \operatorname{diag}\left(m^{\nu}\right)\left(U^{\nu}\right)^{T} \\
& \sim \frac{v_{\mathrm{EW}}^{2}}{M}\left(\begin{array}{ccc}
1 & \epsilon^{q_{L_{1}}-q_{L_{2}}} & \epsilon^{q_{L_{1}}-q_{L_{3}}} \\
\epsilon^{q_{L_{1}}-q_{L_{2}}} & 1 & \epsilon^{q_{L_{2}}-q_{L_{3}}} \\
\epsilon^{q_{L_{1}}-q_{L_{3}}} & \epsilon^{q_{L_{2}}-q_{L_{3}}} & 1
\end{array}\right)\left(\begin{array}{ccc}
0 & 0 & 0 \\
0 & \epsilon^{2 q_{L_{2}}} & 0 \\
0 & 0 & \epsilon^{2 q_{L_{3}}}
\end{array}\right)\left(\begin{array}{ccc}
1 & \epsilon^{q_{L_{1}}-q_{L_{2}}} & \epsilon^{q_{L_{1}}-q_{L_{3}}} \\
\epsilon^{q_{L_{1}}-q_{L_{2}}} & 1 & \epsilon^{q_{L_{2}}-q_{L_{3}}} \\
\epsilon^{q_{L_{1}}-q_{L_{3}}} & \epsilon^{q_{L_{2}}-q_{L_{3}}} & 1
\end{array}\right) .
\end{aligned}
$$

The MNS matrix is given by

$$
U_{\mathrm{MNS}}=U^{\nu} V^{l \dagger} \sim\left(\begin{array}{ccc}
1 & \epsilon^{q_{L_{1}}-q_{L_{2}}} & \epsilon^{q_{L_{1}}-q_{L_{3}}} \\
\epsilon^{q_{L_{1}}-q_{L_{2}}} & 1 & \epsilon^{q_{L_{2}}-q_{L_{3}}} \\
\epsilon^{q_{L_{1}}-q_{L_{3}}} & \epsilon^{q_{L_{2}}-q_{L_{3}}} & 1
\end{array}\right)
$$


Therefore, the large $\nu_{\mu}-\nu_{\tau}$ mixing is obtained for $q_{L_{2}}=q_{L_{3}}$. A reasonable choice to reproduce the observed MNS matrix is thus

$$
\left(\begin{array}{lll}
q_{L_{1}} & q_{L_{2}} & q_{L_{3}}
\end{array}\right)=\left(\begin{array}{lll}
q_{L_{3}}+1 & q_{L_{3}} & q_{L_{3}}
\end{array}\right) .
$$

For $M \sim 10^{14}-10^{15} \mathrm{GeV}$ as a representative value as described in the main text, the observed neutrino mass differences are consistent with $q_{L_{3}}=0$. This is the one given in (2.10). For $M \sim 10^{16}-10^{17} \mathrm{GeV}$, a slightly small Yukawa coupling $y^{N} \sim O(0.01)$ is required. Note that if $q_{L_{3}}$ takes a half-integer value, all the lepton and right-handed neutrino charges should also be half-integer.

Next, let us consider the case of three right-handed neutrinos: $N_{\alpha}(\alpha=1-3)$. For general $\mathrm{U}(1)_{F}$ charge assignments on right-handed neutrinos $\left(q_{N_{1}} q_{N_{2}} q_{N_{3}}\right)$, the Dirac- and Majorana-mass matrices of neutrinos are given by

$$
\begin{gathered}
\left(m_{D}^{\nu}\right)_{i \alpha} \sim v_{\mathrm{EW}}\left(\begin{array}{ccc}
\epsilon^{q_{L_{1}}-q_{N_{1}}} & \epsilon^{q_{L_{1}}-q_{N_{2}}} & \epsilon^{q_{L_{1}}-q_{N_{3}}} \\
\epsilon^{q_{L_{2}}-q_{N_{1}}} & \epsilon^{q_{L_{2}}-q_{N_{2}}} & \epsilon^{q_{L_{2}}-q_{N_{3}}} \\
\epsilon^{q_{L_{3}}-q_{N_{1}}} & \epsilon^{q_{L_{3}}-q_{N_{2}}} & \epsilon^{q_{L_{3}}-q_{N_{3}}}
\end{array}\right), \\
\left(m^{N}\right)_{\alpha \beta} \sim M\left(\begin{array}{ccc}
\epsilon^{-2 q_{N_{1}}} & \epsilon^{-q_{N_{1}}-q_{N_{2}}} & \epsilon^{-q_{N_{1}}-q_{N_{3}}} \\
\epsilon^{-q_{N_{1}}-q_{N_{2}}} & \epsilon^{-2 q_{N_{2}}} & \epsilon^{-q_{N_{2}}-q_{N_{3}}} \\
\epsilon^{-q_{N_{1}}-q_{N_{3}}} & \epsilon^{-q_{N_{2}}-q_{N_{3}}} & \epsilon^{-2 q_{N_{3}}}
\end{array}\right) .
\end{gathered}
$$

The resulting structure of the light neutrino mass matrix after integrating out the heavy right-handed neutrinos is the same as (A.12). The MNS matrix is also the same as (A.14). Only the difference is that there is no zero mass eigenvalues in the light neutrino mass matrix:

$$
\begin{aligned}
m_{i j}^{\nu} & \sim U^{\nu} \operatorname{diag}\left(m^{\nu}\right)\left(U^{\nu}\right)^{T} \\
& \sim \frac{v_{\mathrm{EW}}^{2}}{M}\left(\begin{array}{ccc}
1 & \epsilon^{q_{L_{1}}-q_{L_{2}}} & \epsilon_{L_{1}-q_{L_{3}}}^{q_{L_{2}}} \\
\epsilon^{q_{L_{1}}-q_{L_{2}}} & 1 & \epsilon^{q_{L_{2}}-q_{L_{3}}} \\
\epsilon^{q_{L_{1}}-q_{L_{3}}} & \epsilon^{q_{L_{2}}-q_{L_{3}}} & 1
\end{array}\right)\left(\begin{array}{ccc}
\epsilon^{2 q_{L_{1}}} & 0 & 0 \\
0 & \epsilon^{2 q_{L_{2}}} & 0 \\
0 & 0 & \epsilon^{2 q_{L_{3}}}
\end{array}\right)\left(\begin{array}{ccc}
1 & \epsilon^{q_{L_{1}}-q_{L_{2}}} & \epsilon^{q_{L_{1}}-q_{L_{3}}} \\
\epsilon^{q_{L_{1}}-q_{L_{2}}} & 1 & \epsilon^{q_{L_{2}}-q_{L_{3}}} \\
\epsilon^{q_{L_{1}}-q_{L_{3}}} & \epsilon^{q_{L_{2}}-q_{L_{3}}} & 1
\end{array}\right) .
\end{aligned}
$$

Open Access. This article is distributed under the terms of the Creative Commons Attribution License (CC-BY 4.0), which permits any use, distribution and reproduction in any medium, provided the original author(s) and source are credited.

\section{References}

[1] C.D. Froggatt and H.B. Nielsen, Hierarchy of quark masses, Cabibbo angles and CP-violation, Nucl. Phys. B 147 (1979) 277 [INSPIRE].

[2] R.D. Peccei and H.R. Quinn, CP conservation in the presence of instantons, Phys. Rev. Lett. 38 (1977) 1440 [INSPIRE].

[3] S. Weinberg, A new light boson?, Phys. Rev. Lett. 40 (1978) 223 [INSPIRE].

[4] F. Wilczek, Problem of strong $p$ and $t$ invariance in the presence of instantons, Phys. Rev. Lett. 40 (1978) 279 [INSPIRE]. 
[5] J. Preskill, M.B. Wise and F. Wilczek, Cosmology of the invisible axion, Phys. Lett. 120B (1983) 127.

[6] L.F. Abbott and P. Sikivie, A cosmological bound on the invisible axion, Phys. Lett. 120B (1983) 133.

[7] M. Dine and W. Fischler, The not so harmless axion, Phys. Lett. 120B (1983) 137.

[8] P. Langacker, R.D. Peccei and T. Yanagida, Invisible axions and light neutrinos: are they connected?, Mod. Phys. Lett. A 1 (1986) 541 [inSPIRE].

[9] T. Yanagida, Horizontal symmetry and masses of neutrinos, Conf. Proc. C 7902131 (1979) 95.

[10] M. Gell-Mann, P. Ramond and R. Slansky, Complex spinors and unified theories, Conf. Proc. C 790927 (1979) 315 [arXiv:1306.4669] [INSPIRE].

[11] P. Minkowski, $\mu \rightarrow$ er at a rate of one out of $10^{9}$ muon decays?, Phys. Lett. B 67 (1977) 421 [INSPIRE].

[12] F. Wilczek, Axions and family symmetry breaking, Phys. Rev. Lett. 49 (1982) 1549 [INSPIRE].

[13] C.Q. Geng and J.N. Ng, Flavor connections and neutrino mass hierarchy invariant invisible axion models without domain wall problem, Phys. Rev. D 39 (1989) 1449 [INSPIRE].

[14] Z.G. Berezhiani and M. Yu. Khlopov, Cosmology of spontaneously broken gauge family symmetry, Z. Phys. C 49 (1991) 73 [InSPIRE].

[15] K.S. Babu and S.M. Barr, Family symmetry, gravity and the strong CP problem, Phys. Lett. B 300 (1993) 367 [hep-ph/9212219] [INSPIRE].

[16] M.E. Albrecht, T. Feldmann and T. Mannel, Goldstone bosons in effective theories with spontaneously broken flavour symmetry, JHEP 10 (2010) 089 [arXiv:1002.4798] [INSPIRE].

[17] C.S. Fong and E. Nardi, Spontaneous breaking of flavor symmetry avoids the strong CP problem, Phys. Rev. Lett. 111 (2013) 061601 [arXiv:1305.1627] [INSPIRE].

[18] Y.H. Ahn, Flavored Peccei-Quinn symmetry, Phys. Rev. D 91 (2015) 056005 [arXiv: 1410.1634] [INSPIRE].

[19] Y.H. Ahn, Flavored Universe dispatched via Axion and Neutrino, arXiv:1611.08359 [INSPIRE].

[20] A. Celis, J. Fuentes-Martin and H. Serodio, An invisible axion model with controlled FCNCs at tree level, Phys. Lett. B 741 (2015) 117 [arXiv:1410.6217] [InSPIRE].

[21] A. Celis, J. Fuentes-Martín and H. Serôdio, A class of invisible axion models with FCNCs at tree level, JHEP 12 (2014) 167 [arXiv:1410.6218] [INSPIRE].

[22] A. Salvio, A simple motivated completion of the standard model below the Planck scale: axions and right-handed neutrinos, Phys. Lett. B 743 (2015) 428 [arXiv:1501.03781] [INSPIRE].

[23] G. Ballesteros, J. Redondo, A. Ringwald and C. Tamarit, Unifying inflation with the axion, dark matter, baryogenesis and the seesaw mechanism, arXiv:1608.05414 [INSPIRE].

[24] G. Ballesteros, J. Redondo, A. Ringwald and C. Tamarit, Standard model-axion-seesaw-Higgs portal inflation. Five problems of particle physics and cosmology solved in one stroke, arXiv: 1610.01639 [INSPIRE]. 
[25] R. Kallosh and A. Linde, Universality class in conformal inflation, JCAP 07 (2013) 002 [arXiv: 1306.5220] [INSPIRE].

[26] S. Ferrara, R. Kallosh, A. Linde and M. Porrati, Minimal supergravity models of inflation, Phys. Rev. D 88 (2013) 085038 [arXiv:1307.7696] [InSPIRE].

[27] R. Kallosh, A. Linde and D. Roest, Superconformal inflationary $\alpha$-attractors, JHEP 11 (2013) 198 [arXiv:1311.0472] [INSPIRE].

[28] M. Galante, R. Kallosh, A. Linde and D. Roest, Unity of cosmological inflation attractors, Phys. Rev. Lett. 114 (2015) 141302 [arXiv:1412.3797] [INSPIRE].

[29] M. Fukugita and T. Yanagida, Baryogenesis without grand unification, Phys. Lett. B 174 (1986) 45 [INSPIRE].

[30] J.E. Kim, Weak interaction singlet and strong CP invariance, Phys. Rev. Lett. 43 (1979) 103 [INSPIRE].

[31] M.A. Shifman, A.I. Vainshtein and V.I. Zakharov, Can confinement ensure natural CP invariance of strong interactions?, Nucl. Phys. B 166 (1980) 493 [INSPIRE].

[32] M. Dine, W. Fischler and M. Srednicki, A simple solution to the strong CP problem with a harmless axion, Phys. Lett. B 104 (1981) 199 [INSPIRE].

[33] A.R. Zhitnitsky, On possible suppression of the axion hadron interactions (in Russian), Sov. J. Nucl. Phys. 31 (1980) 260 [Yad. Fiz. 31 (1980) 497] [INSPIRE].

[34] P.H. Frampton, S.L. Glashow and T. Yanagida, Cosmological sign of neutrino CP-violation, Phys. Lett. B 548 (2002) 119 [hep-ph/0208157] [INSPIRE].

[35] A. Ibarra and G.G. Ross, Neutrino phenomenology: the case of two right-handed neutrinos, Phys. Lett. B 591 (2004) 285 [hep-ph/0312138] [INSPIRE].

[36] T. Yanagida and J. Sato, Large lepton mixing in seesaw models: coset space family unification, Nucl. Phys. Proc. Suppl. 77 (1999) 293 [hep-ph/9809307] [InSPIRE].

[37] J. Sato and T. Yanagida, Low-energy predictions of lopsided family charges, Phys. Lett. B 493 (2000) 356 [hep-ph/0009205] [INSPIRE].

[38] P. Ramond, Neutrinos: a glimpse beyond the standard model, Nucl. Phys. Proc. Suppl. 77 (1999) 3 [hep-ph/9809401] [INSPIRE].

[39] J.E. Kim, Light pseudoscalars, particle physics and cosmology, Phys. Rept. 150 (1987) 1 [INSPIRE].

[40] P. Sikivie, Experimental tests of the invisible axion, Phys. Rev. Lett. 51 (1983) 1415 [Erratum ibid. 52 (1984) 695] [INSPIRE].

[41] R. Bradley et al., Microwave cavity searches for dark-matter axions, Rev. Mod. Phys. 75 (2003) 777 [INSPIRE].

[42] P.W. Graham and S. Rajendran, New observables for direct detection of axion dark matter, Phys. Rev. D 88 (2013) 035023 [arXiv: 1306.6088] [inSPIRE].

[43] D. Budker, P.W. Graham, M. Ledbetter, S. Rajendran and A. Sushkov, Proposal for a Cosmic Axion Spin Precession Experiment (CASPEr), Phys. Rev. X 4 (2014) 021030 [arXiv: 1306.6089] [INSPIRE].

[44] E787 and E949 collaborations, S. Adler et al., Measurement of the $K^{+} \rightarrow \pi^{+} \nu \nu$ branching ratio, Phys. Rev. D 77 (2008) 052003 [arXiv:0709.1000] [INSPIRE]. 
[45] NA62 collaboration, M. Moulson, Search for $K^{+} \rightarrow \pi^{+} \nu \bar{\nu}$ at NA62, arXiv:1611.04979 [INSPIRE].

[46] J.T. Goldman et al., Light boson emission in the decay of the $\mu^{+}$, Phys. Rev. D 36 (1987) 1543 [INSPIRE].

[47] R.D. Bolton et al., Search for rare muon decays with the crystal box detector, Phys. Rev. D 38 (1988) 2077 [INSPIRE].

[48] J.L. Feng, T. Moroi, H. Murayama and E. Schnapka, Third generation familons, $b$ factories and neutrino cosmology, Phys. Rev. D 57 (1998) 5875 [hep-ph/9709411] [inSPIRE].

[49] I. Dorsner and S.M. Barr, Flavor exchange effects in models with Abelian flavor symmetry, Phys. Rev. D 65 (2002) 095004 [hep-ph/0201207] [INSPIRE].

[50] K. Tsumura and L. Velasco-Sevilla, Phenomenology of flavon fields at the LHC, Phys. Rev. D 81 (2010) 036012 [arXiv:0911.2149] [INSPIRE].

[51] K. Huitu, V. Keus, N. Koivunen and O. Lebedev, Higgs-flavon mixing and $h \rightarrow \mu \tau$, JHEP 05 (2015) 026 [arXiv: 1603.06614] [INSPIRE].

[52] M. Bauer, T. Schell and T. Plehn, Hunting the flavon, Phys. Rev. D 94 (2016) 056003 [arXiv: 1603.06950] [INSPIRE].

[53] G.G. Raffelt, Stars as laboratories for fundamental physics: the astrophysics of neutrinos, axions, and other weakly interacting particles, Chicago University Press, Chicago U.S.A. (1996).

[54] G.G. Raffelt, Astrophysical axion bounds, Lect. Notes Phys. 741 (2008) 51 [hep-ph/0611350] [INSPIRE].

[55] M.M. Miller Bertolami, B.E. Melendez, L.G. Althaus and J. Isern, Revisiting the axion bounds from the Galactic white dwarf luminosity function, JCAP 10 (2014) 069 [arXiv: 1406.7712] [INSPIRE].

[56] S. Weinberg, Baryon and lepton nonconserving processes, Phys. Rev. Lett. 43 (1979) 1566 [INSPIRE].

[57] P. Nath and P. Fileviez Perez, Proton stability in grand unified theories, in strings and in branes, Phys. Rept. 441 (2007) 191 [hep-ph/0601023] [INSPIRE].

[58] Super-Kamiokande collaboration, V. Takhistov, Review of nucleon decay searches at Super-Kamiokande, arXiv:1605.03235 [INSPIRE].

[59] M. Kawasaki and K. Nakayama, Axions: theory and cosmological role, Ann. Rev. Nucl. Part. Sci. 63 (2013) 69 [arXiv: 1301.1123] [INSPIRE].

[60] M.S. Turner, Cosmic and local mass density of invisible axions, Phys. Rev. D 33 (1986) 889 [INSPIRE].

[61] Planck collaboration, P.A.R. Ade et al., Planck 2015 results. XX. Constraints on inflation, Astron. Astrophys. 594 (2016) A20 [arXiv:1502.02114] [INSPIRE].

[62] S. Antusch, S.F. King, M. Malinsky, L. Velasco-Sevilla and I. Zavala, Flavon inflation, Phys. Lett. B 666 (2008) 176 [arXiv: 0805.0325] [INSPIRE].

[63] L. Kofman, A.D. Linde and A.A. Starobinsky, Nonthermal phase transitions after inflation, Phys. Rev. Lett. 76 (1996) 1011 [hep-th/9510119] [INSPIRE]. 
[64] S. Kasuya, M. Kawasaki and T. Yanagida, Cosmological axion problem in chaotic inflationary universe, Phys. Lett. B 409 (1997) 94 [hep-ph/9608405] [INSPIRE].

[65] S. Kasuya, M. Kawasaki and T. Yanagida, Domain wall problem of axion and isocurvature fluctuations in chaotic inflation models, Phys. Lett. B 415 (1997) 117 [hep-ph/9709202] [INSPIRE].

[66] M. Kawasaki, T.T. Yanagida and K. Yoshino, Domain wall and isocurvature perturbation problems in axion models, JCAP 11 (2013) 030 [arXiv:1305.5338] [INSPIRE].

[67] F. Takahashi, Linear inflation from running kinetic term in supergravity, Phys. Lett. B 693 (2010) 140 [arXiv: 1006.2801] [INSPIRE].

[68] K. Nakayama and F. Takahashi, Running kinetic inflation, JCAP 11 (2010) 009 [arXiv: 1008.2956] [INSPIRE].

[69] A.R. Liddle and D.H. Lyth, Cosmological inflation and large scale structure, Cambridge University Press, Cambridge U.K. (2000).

[70] M. Herranen, T. Markkanen, S. Nurmi and A. Rajantie, Spacetime curvature and Higgs stability after inflation, Phys. Rev. Lett. 115 (2015) 241301 [arXiv:1506. 04065] [INSPIRE].

[71] Y. Ema, K. Mukaida and K. Nakayama, Fate of electroweak vacuum during preheating, JCAP 10 (2016) 043 [arXiv: 1602.00483] [INSPIRE].

[72] K. Kohri and H. Matsui, Higgs vacuum metastability in primordial inflation, preheating and reheating, Phys. Rev. D 94 (2016) 103509 [arXiv:1602.02100] [InSPIRE].

[73] K. Enqvist, M. Karciauskas, O. Lebedev, S. Rusak and M. Zatta, Postinflationary vacuum instability and Higgs-inflaton couplings, JCAP 11 (2016) 025 [arXiv:1608.08848] [INSPIRE].

[74] O. Lebedev, On stability of the electroweak vacuum and the Higgs portal, Eur. Phys. J. C 72 (2012) 2058 [arXiv: 1203.0156] [INSPIRE].

[75] J. Elias-Miro, J.R. Espinosa, G.F. Giudice, H.M. Lee and A. Strumia, Stabilization of the electroweak vacuum by a scalar threshold effect, JHEP 06 (2012) 031 [arXiv:1203.0237] [INSPIRE].

[76] W. Buchmüller, P. Di Bari and M. Plümacher, Leptogenesis for pedestrians, Annals Phys. 315 (2005) 305 [hep-ph/0401240] [INSPIRE].

[77] K. Hamaguchi, H. Murayama and T. Yanagida, Leptogenesis from $N$ dominated early universe, Phys. Rev. D 65 (2002) 043512 [hep-ph/0109030] [InSPIRE].

[78] S. Davidson and A. Ibarra, A lower bound on the right-handed neutrino mass from leptogenesis, Phys. Lett. B 535 (2002) 25 [hep-ph/0202239] [INSPIRE].

[79] L. Covi and E. Roulet, Baryogenesis from mixed particle decays, Phys. Lett. B 399 (1997) 113 [hep-ph/9611425] [INSPIRE].

[80] A. Pilaftsis, Resonant CP-violation induced by particle mixing in transition amplitudes, Nucl. Phys. B 504 (1997) 61 [hep-ph/9702393] [INSPIRE].

[81] W. Buchmüller and M. Plümacher, CP asymmetry in Majorana neutrino decays, Phys. Lett. B 431 (1998) 354 [hep-ph/9710460] [INSPIRE].

[82] A. Pilaftsis and T.E.J. Underwood, Resonant leptogenesis, Nucl. Phys. B 692 (2004) 303 [hep-ph/0309342] [INSPIRE]. 
[83] M. Garny, A. Kartavtsev and A. Hohenegger, Leptogenesis from first principles in the resonant regime, Annals Phys. 328 (2013) 26 [arXiv:1112.6428] [INSPIRE].

[84] A. Abada, S. Davidson, F.-X. Josse-Michaux, M. Losada and A. Riotto, Flavor issues in leptogenesis, JCAP 04 (2006) 004 [hep-ph/0601083] [INSPIRE].

[85] E. Nardi, Y. Nir, E. Roulet and J. Racker, The importance of flavor in leptogenesis, JHEP 01 (2006) 164 [hep-ph/0601084] [INSPIRE].

[86] A. Abada, S. Davidson, A. Ibarra, F.X. Josse-Michaux, M. Losada and A. Riotto, Flavour matters in leptogenesis, JHEP 09 (2006) 010 [hep-ph/0605281] [INSPIRE].

[87] G.C. Branco, P.M. Ferreira, L. Lavoura, M.N. Rebelo, M. Sher and J.P. Silva, Theory and phenomenology of two-Higgs-doublet models, Phys. Rept. 516 (2012) 1 [arXiv:1106.0034] [INSPIRE].

[88] M. Kawasaki, K. Saikawa and T. Sekiguchi, Axion dark matter from topological defects, Phys. Rev. D 91 (2015) 065014 [arXiv: 1412.0789] [INSPIRE]. 